CONGENITAL HEART DISEASE

\title{
The role of echocardiography in diagnosing double chambered right ventricle in adults
}

\author{
P Hoffman, A W Wójcik, J Różański, H Siudalska, E Jakubowska, E K Włodarska, M Kowalski
}

Heart 2004;90:789-793. doi: 10.1136/hrt.2003.017137

See end of article for authors' affiliations

.....................

Correspondence to: Dr Anna Wójcik, National Institute of Cardiology, Department of Congenita Heart Diseases, 04-628

Warszawa, Alpejska 42,

Poland; awojcik@ ikard.waw.pl

Accepted 16 October 2003

\begin{abstract}
Objective: To evaluate the utility of echocardiography in diagnosing double chambered right ventricle (DCRV) in adults.

Design: A retrospective study.

Setting: Department of Congenital Heart Diseases and Department of Cardiac Surgery, Institute of Cardiology, Warsaw, Poland.

Patients: 32 patients, mean age 32.5 years, 21 female, and 11 male.

Intervention: Transthoracic (TTE) and transoesophageal (TOE) echocardiographic examination in patients with suspected DCRV.

Main outcome measures: Direct inspection during surgical treatment of 28 patients diagnosed as having DCRV as an isolated lesion or associated with other pathologies.

Results: Echocardiography allowed the final diagnosis of DCRV in 26 patients (81\%) out of 32 studied. TTE was diagnostic in $5(15.6 \%)$ whereas TOE was diagnostic in 21 of 21 studied by this technique. Of 6 patients with negative $\Pi E, D C R V$ was identified by cardiac catheterisation in 3 and directly during surgery in the remaining 3 . Of 26 patients diagnosed by echocardiography, the anomalous muscle bundle was discrete in $20(77 \%)$ and diffuse in $6(23 \%)$. In 23 patients (88\%) right ventricular oufflow obstruction was localised low in the right ventricle, and in the remaining $3(11.5 \%)$, the obstruction was localised high in the right ventricle. Abnormal bundles localised high were discrete, bundles localised in the lower part of right ventricle were discrete in $17(74 \%)$ and diffuse in the remaining $6(26 \%)$. DCRV was an isolated lesion in only 2 patients $(6.2 \%)$. In all subjects surgical inspection confirmed echocardiographic data.

Conclusions: Echocardiography was very useful to diagnose DCRV in adults as well as to identify its anatomic type. Comparing two different approaches, TOE better defines the entire scope of pathology, including estimation of the resultant systolic pressure gradient within the right ventricular cavity.
\end{abstract}

D ouble chambered right ventricle (DCRV) is a congenital heart defect where the right ventricle is divided by anomalous muscle bundle into a high pressure inlet portion and a low pressure outlet portion. It is seen in only $0.5-2 \%$ of all cases of congenital heart disease. Localisation of the obstructive muscle varies and may be either high (or horizontal), adjacent to the pulmonary valve, or low (or oblique), close to the apex. ${ }^{1}$ It may also develop over time as an acquired lesion in patients with an abnormally short distance between the moderator band and the pulmonary valve. $^{2}$

It has also been suggested that the increased blood flow and pressure within the right ventricular outflow tract may act as an initial stimulus for hypertrophy of the crista supraventricularis in patients with ventricular septal defect (VSD) and notably increased pulmonary blood flow. ${ }^{3}$ The resulting obstruction usually deteriorates, with subsequent hypertrophy of the muscle and further narrowing of the right ventricular cavity. Such a complication has even been observed in cases with stable narrowing. ${ }^{4}$

DCRV is exceptionally rare as an isolated anomaly. Most commonly (in around 90\%) it is associated with a membranous type VSD. The other coexisting lesions include subaortic stenosis, pulmonary valve stenosis, double outlet right ventricle, tetralogy of Fallot, anomalous pulmonary venous drainage, complete or corrected transposition of the great arteries, pulmonary atresia with intact ventricular septum, and Ebstein anomaly. ${ }^{4}$

Although transthoracic echocardiography (TTE) is widely accepted as a method of demonstrating DCRV in paediatric patients, substantial problems may arise while studying adults. Implementation of the transoesophageal technique (TOE) allows better definition of the lesion in adults. In some cases, other modes, including magnetic resonance or cardiac catheterisation, may be required..$^{4-6}$

The aim of our study was to define the relative value of TTE and TOE in diagnosing various forms of DCRV in adults.

\section{METHODS}

We carried out a retrospective review of 32 patients who had been admitted to the department of congenital heart diseases, Institute of Cardiology, Warsaw, between 1989 and 2001 and in whom DCRV had been diagnosed. Twenty one were female $(66 \%)$ and 11 were male $(34 \%)$. Their mean age was 32.5 years (range 16-53 years). TTE had been done in all these patients and was analysed for its ability to define the morphology of the right ventricle and an anomalous bundle. The latter was labelled as high, if localised adjacent to the pulmonary valve (fig 1), or low, if localised close to the apex (fig 2). In addition, obstruction was described as diffuse if its shape was triangular and its attachment to the right ventricular wall was long (fig 2), or discrete if the attachment of its base to the right ventricular wall was short and well defined (figs 1 and 3).

Abbreviations: DCRV, double chambered right ventricle; TOE, transoesophageal echocardiography; TTE, transthoracic echocardiography; VSD, ventricular septal defect 


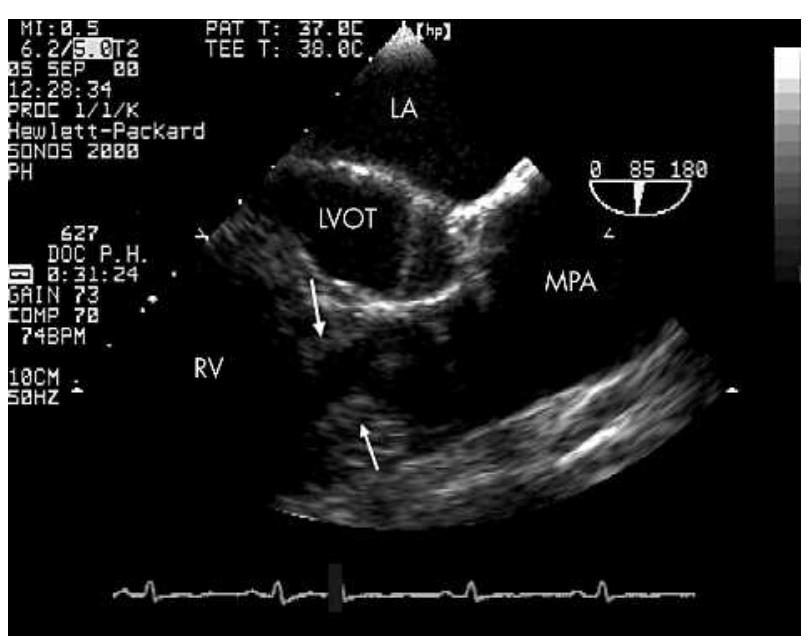

Figure 1 Double chambered right ventricle (DCRV). High and discrete obstruction of the right ventricular cavity (arrows) adjacent to the pulmonary valve, demonstrated by transoesophageal echocardiography in the longitudinal plane $\left(85^{\circ}\right)$. In this case DCRV is associated with valvar pulmonary stenosis. LA, left atrium; LVOT, left ventricular oufflow tract; MPA, main pulmonary artery; RV, right ventricle.

Where transthoracic imaging was equivocal or uninformative, TOE was done. Both transoesophageal and transgastric views were used to define the anatomy of the right ventricle.

The echocardiographic study was completed by evaluation of the resultant systolic pressure gradient within the right ventricle using continuous wave Doppler. Interrogation was guided by colour Doppler so as to define the exact site of the obstruction by the appearance of a mosaic pattern where the high velocity flow originated.

DCRV was diagnosed if an obstruction was clearly demonstrated and classified morphologically, and there was a midcavity pressure gradient proven by continuous wave Doppler, or abnormal acceleration of the flow within the right ventricle shown by colour Doppler, or both (figs 4 and 5). The echocardiographic examination was considered definitive if it showed the overall pathology of the heart and allowed further clinical decision making. In cases of persisting doubt, other diagnostic modes were used.

\section{RESULTS}

Of the 32 patients, only five were diagnosed solely by means of TTE (15.6\%) (fig 6). TOE demonstrated DCRV in all 21 patients in whom the study was done. Of the six patients with negative TTE, DCRV was found in three by cardiac catheterisation and in the remaining three by direct inspection during surgery carried out for other reasons.

In three of the five patients in whom TTE was sufficient to demonstrate DCRV, subcostal images were the most useful (transverse plane in two and frontal plane in one), while in the remaining two patients parasternal short axis planes were of superior value.

Twenty one patients required subsequent TOE for evaluating DCRV. The monoplane technique was applied in five cases while omniplane TOE, available in our department since 1993, was done in the remaining 16.

In three patients DCRV was discovered during cardiac catheterisation, which was necessary in those cases because of lack of a TOE probe. Three cases of DCRV were detected during surgical correction of the other disorders.

In the 26 patients diagnosed by echocardiography, the anomalous muscle bundle was discrete in 20 (77\%) and diffuse in six $(23 \%)$. In 23 patients $(88 \%)$, right ventricular outflow obstruction was low and in the remaining three

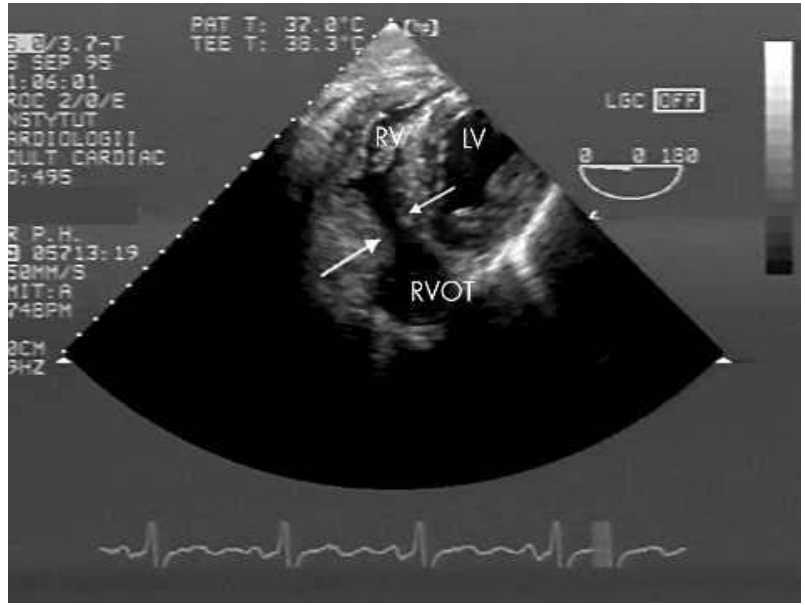

Figure 2 Double chambered right ventricle. Low and diffuse obstruction of the right ventricular cavity (arrows), demonstrated by transoesophageal echocardiography from within the fundus of the stomach in the transverse plane $\left(0^{\circ}\right)$. LV, left ventricle; RV, right ventricle; RVOT, right ventricular oufflow tract.

(11.5\%) it was high. High localisation was associated with the discrete form of obstruction, whereas a low abnormal bundle was discrete in 17 and diffuse in the remaining six.

Maximum systolic pressure gradient within the right ventricle ranged from 20 to $135 \mathrm{~mm} \mathrm{Hg}$ and exceeded $50 \mathrm{~mm} \mathrm{Hg}$ in 19 cases (73\%). TTE allowed gradient estimation by continuous wave Doppler in 11 patients (the morphology, however, could only be fully described in five); in 13 patients this was also achieved by TOE when transgastric imaging was applied. In some cases, however, maximum flow velocity could be evaluated only in a single cycle despite extensive probe manipulation (fig 5). In the remaining six patients, assessment of the right ventricular gradient during TOE was not attempted, as its value was known from TTE. In two patients, lack of proper beam alignment precluded its measurement.

There were two patients in whom DCRV was an isolated anomaly $(6.2 \%)$. Twenty seven had a coexisting VSD $(84.4 \%)$ and 10 had discrete subaortic stenosis (31.25\%). Table 1 shows all the lesions associated with DCRV in this cohort.

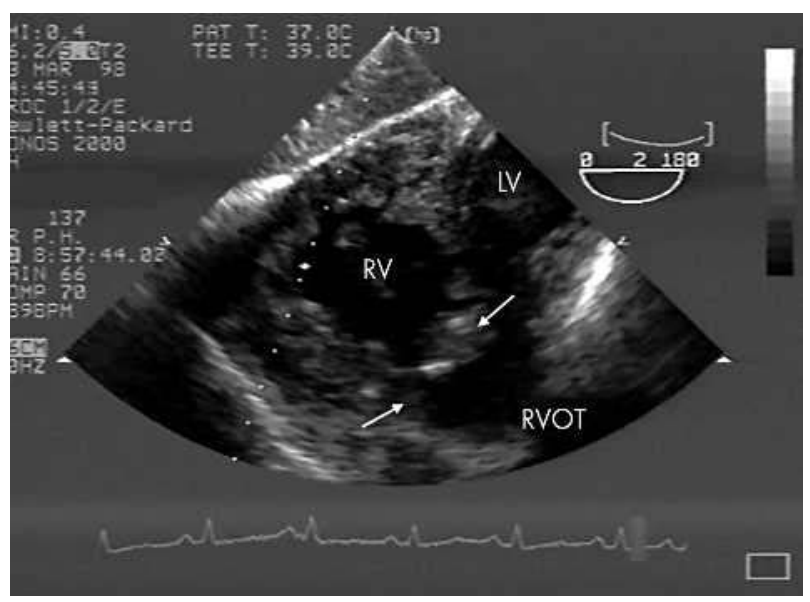

Figure 3 Double chambered right ventricle, low and discrete obstruction (arrows) demonstrated by transoesophageal echocardiography from within the fundus of the stomach in the horizontal plane $\left(0^{\circ}\right)$. LV, left ventricle; RV, right ventricle; RVOT, right ventricular oufflow tract. 

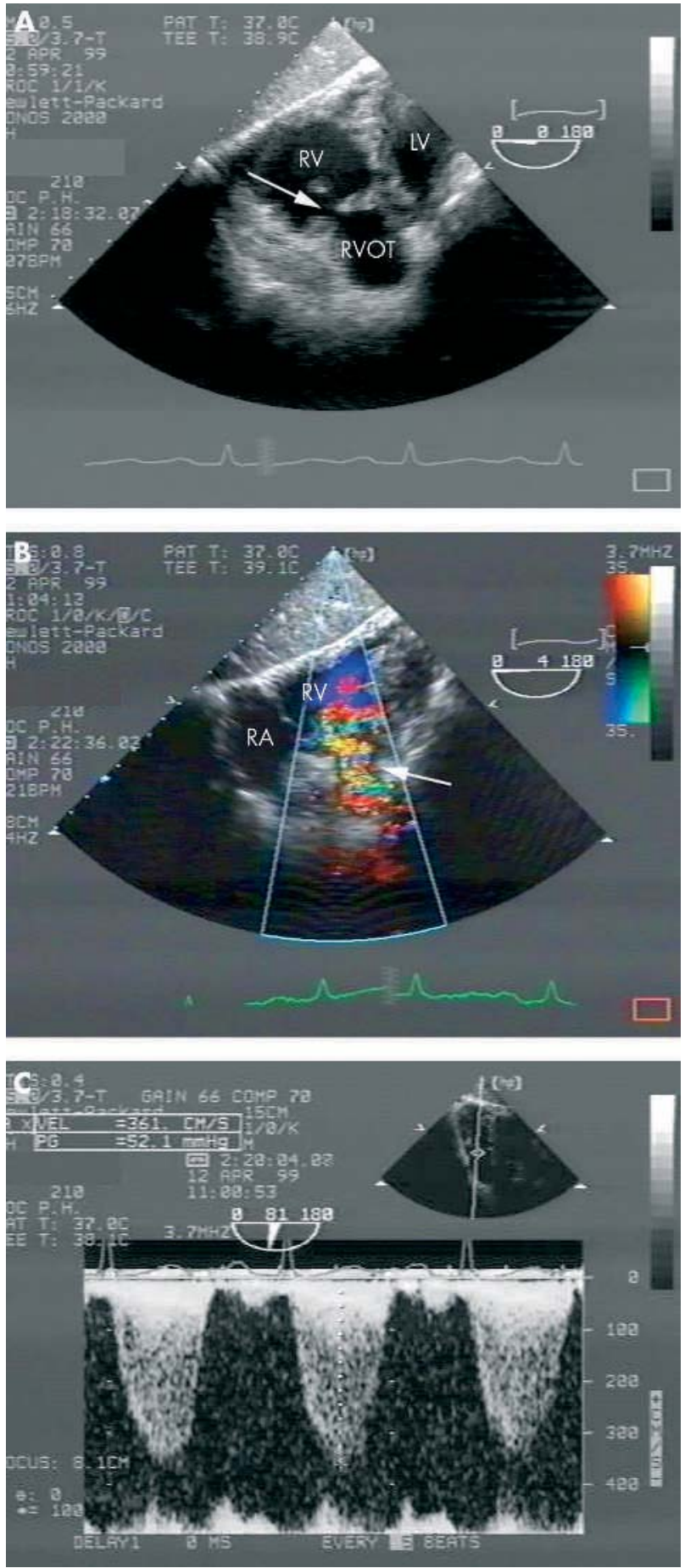

Figure 4 (A) Double chambered right ventricle with low and discrete obstruction (arrow). Transoesophageal echocardiography from within the fundus of the stomach in the horizontal plane $\left(0^{\circ}\right)$. (B) Same study plane, frontal view of the entire right ventricle, end systolic frame. Colour coded flow Doppler imaging indicates origin of the turbulent flow within the right ventricular cavity (arrow). (C) Colour coded Doppler echocardiography allows proper quantitative evaluation of the mid cavitary pressure gradient of $52.1 \mathrm{~mm} \mathrm{Hg}$ by continuous wave Doppler. This also required rotation of the studying plane $\left(81^{\circ}\right)$. LV, left ventricle RA, right atrium; RV, right ventricle; RVOT, right ventricular oufflow tract.

Twenty eight patients underwent surgical treatment, including incision of the anomalous muscular band within the right ventricle and correction of the associated anomalies. In all cases surgical inspection confirmed the echocardiographic data. There were no hospital or late deaths.

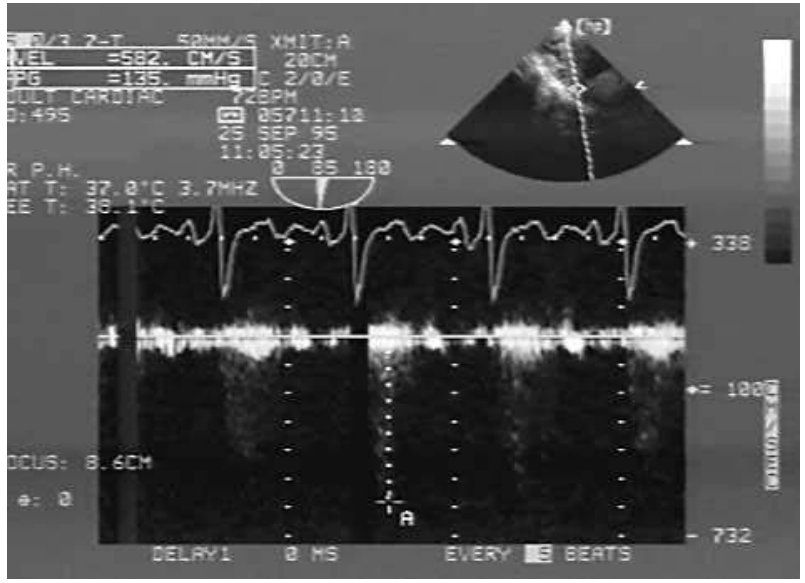

Figure 5 Double chambered right ventricle. continuous wave Doppler revealed midcavitary pressure gradient of $135 \mathrm{~mm} \mathrm{Hg}$ detected in a single evolution. Transoesophageal echocardiography from the fundus of the stomach.

Postoperative right ventricular pressure gradient did not exceed $23 \mathrm{~mm} \mathrm{Hg}$ in any case except for one, in whom a gradient of $62 \mathrm{~mm} \mathrm{Hg}$ remained.

\section{DISCUSSION}

Double chambered right ventricle is a rare form of congenital heart disease in which the right ventricle is divided into a high pressure inlet portion and a low pressure outlet portion by an anomalous muscle bundle. ${ }^{1}$ DCRV was originally described more than 130 years ago, but it has only been reported extensively in clinical series since the 1960s. As outlined by Restivo and colleagues, ${ }^{7}$ there are several subtypes of divided right ventricle. These may involve the following: an anomalous septoparietal band; an anomalous apical shelf; an anomalous apical shelf with Ebstein malformation; an apical shelf confluent with an outlet septum; a sequestrated ventricular inlet portion; a sequestrated trabecular component; or sequestration of the outlet portion. ${ }^{47}$ A contemporary analysis of the origin of the muscle bundles determined that the muscular shelf originates from the body of the septomarginal trabeculation. A simple classification of the pathology was proposed by Folger,

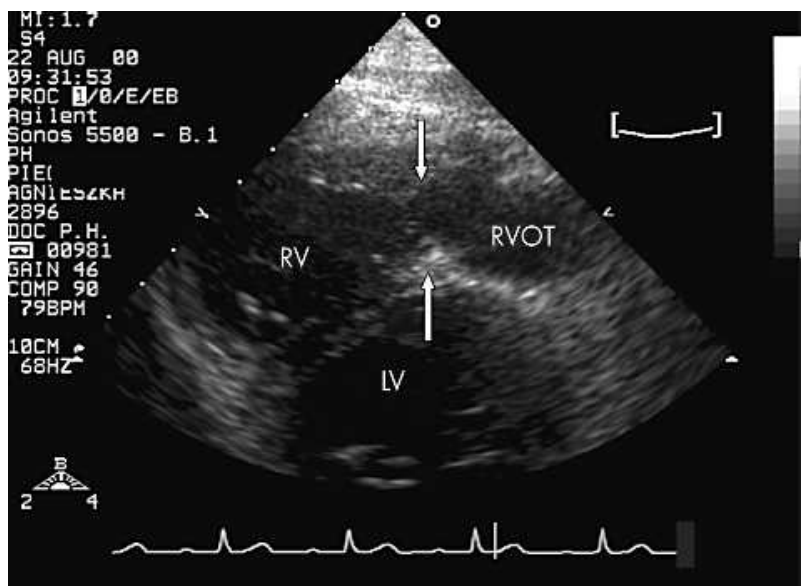

Figure 6 Double chambered right ventricle. Transthoracic echocardiography. Short parasternal view shows diffuse low obstruction (arrow). LV, left ventricle; RV, right ventricle; RVOT, right ventricular oufflow tract. 


\begin{tabular}{ll}
$\begin{array}{l}\text { Table } 1 \\
\text { ventricle }\end{array}$ & Lesions associated with double chambered right \\
\hline $\begin{array}{l}\text { No of } \\
\text { patients }\end{array}$ & Cardiac anomalies \\
\hline 2 & Isolated anomaly \\
15 & VSD \\
1 & VSD, aortic insufficiency \\
2 & VSD, PFO \\
1 & VSD, PS valvar pulmonary stenosis \\
1 & Aneurys of IVS \\
4 & VSD, SAOM \\
1 & VSD, SAOM, ASD II \\
1 & VSD, SAOM, PFO \\
1 & VSD, SAOM, aortic insufficiency \\
1 & VSD, SAOM, aortic and mitral insufficiency \\
1 & SAOM \\
1 & SAOM, CoA, aortic and mitral insufficiency
\end{tabular}

ASD, atrial septal defect; IVS, interventricular septum; PFO, patent foramen ovale; PS, valvar pulmonary stenosis; SAoM, membranous subaortic stenosis; VSD, ventricular septal defect.

who described two positions of the abnormal muscle bundle: high (or horizontal) and low (or oblique). ${ }^{8}$

The treatment of the DCRV is surgical and most often constitutes a part of the corrective procedure. The time for intervention usually depends on the associated lesions. In the absence of a significant coexisting defect, observation may be appropriate as long as the intracavitary systolic gradient is not greater than $40 \mathrm{~mm} \mathrm{Hg}$ and the obstruction is not progressive. The long term results of surgical treatment are excellent. ${ }^{49}{ }^{10}$

In our study we assessed echocardiography as the definitive diagnostic tool in defining the right ventricular obstruction in adults. Transthoracic scanning, although very accurate for paediatric patients, was fully successful in only five of our adult patients. This reflected problems with delineating the morphology of the right ventricular outflow tract from precordial planes owing to its proximity to the transducer, and other limitations encountered while studying adults, such as obesity and emphysema. If available, the subcostal plane was the most diagnostic. In six patients, an abnormal pressure gradient within the right ventricle suggested obstruction. TOE was much more rewarding and allowed a diagnosis of DCRV and coexisting lesions in all 21 subjects studied by this means. Monoplane examination, however, always required deep transgastric imaging as the right ventricular outflow tract is poorly visualised from within the oesophagus, in contrast to the omniplane approach. ${ }^{11}$

When both techniques were used in combination, a definitive diagnosis of DCRV could be established in 26 patients $(81.25 \%)$. In another three, the lesion was revealed by cardiac catheterisation, which was employed when TOE was not available in our institution. In the final three patients, DCRV was found unexpectedly during surgery. In the first of these, surgery was carried out because of a large perimembranous VSD coexisting with an obstructive subaortic membrane and severe aortic regurgitation; in the second, because of a perimembranous septal defect; and in the third, because of severe mitral and aortic regurgitation.

Echocardiography allowed anatomical classification of the abnormal muscle bands that resulted in the right ventricular obstruction. The discrete form of the obstruction was more common than the diffuse (20 v 6 patients), while low localisation was more common than high (23 v 3). The maximum systolic pressure gradient between the inflow and outflow chamber ranged between $20-135 \mathrm{~mm} \mathrm{Hg}$ and exceeded $50 \mathrm{~mm} \mathrm{Hg}$ in 19 cases (59.4\%). Both TTE and TOE (particularly with the use of transgastric planes) were helpful in measuring pressure gradients.
In 22 of the 28 patients who were subsequently operated on, echocardiography was definitive in reaching a final diagnosis and in clinical decision making. Three patients were operated on solely on the basis of invasive studies, and three following direct surgical inspection.

Our study confirms that DCRV is rarely found as an isolated anomaly. Associated defects were present in approximately $80-90 \%$ of patients, a perimembranous VSD being the most common. In our group, the most frequent associated anomaly was VSD $(84.4 \%)$, followed by membranous subaortic stenosis (31.25\%). As far as we are aware, the largest series of DCRV diagnosed echocardiographically in adults was recently published by Lascano and colleagues. ${ }^{1}$ In their experience also, TOE was an excellent technique for demonstrating the lesion. TTE was of limited value but worked better in the context of a congenital echocardiography laboratory. Interestingly, cardiac catheterisation failed in three patients. The authors emphasised that both echocardiography and right sided cardiac catheterisation should be interpreted by personnel with experience in congenital heart disease, to increase the likelihood of a correct diagnosis.

Another method that may be used successfully for evaluating DCRV is magnetic resonance, although experience with this technique is still limited. The images obtained, however, allow precise definition of the exact nature of the right ventricular obstruction. ${ }^{6}$

Most cases of DCRV are discovered during childhood by means of TTE and, rarely, TOE. ${ }^{12}$ Thus the lesion is only occasionally seen in adults. One needs to be aware that its association with VSD may be a source of clinical misinterpretation as a small VSD with a left to right shunt may give rise to both a systolic thrill and a harsh murmur easily audible along left sternal border. As TTE is often insufficient to demonstrate DCRV, TOE is strongly advised for both children and adults to avoid missing the diagnosis, particularly in the presence of right ventricular hypertrophy on ECG. The severity of the obstruction can be assessed by continuous wave Doppler examination of the obstructed region. The exact site of obstruction should be identified on colour flow Doppler by the appearance of a mosaic pattern where the high velocity flow originates. ${ }^{9}$

\section{Conclusions}

DCRV is a rare congenital cardiac anomaly in adults. Echocardiography is very useful in diagnosing the anomaly in adult patients. TOE, however, is much more capable than TTE of defining the full details of the pathology, including estimation of the pressure gradient. The abnormal muscle bundle is more often localised low than high within the right ventricle. High localisation of the abnormal bundle is invariably associated with discrete morphology, whereas a low obstruction may be either discrete or diffuse, the latter being less common. DCRV is usually associated with a VSD or, less often, with membranous subaortic obstruction. Surgical treatment carries a low risk and provides excellent immediate results in these patients.

\section{Authors' affiliations \\ P Hoffman, A W Wójcik, H Siudalska, E Jakubowska, E K Włodarska, M Kowalski, Department of Congenital Heart Diseases, Institute of Cardiology, Warsaw, Poland \\ J Różański, Department of Cardiac Surgery, Institute of Cardiology, Warsaw}

\section{REFERENCES}

1 Lascano ME, Schaad MS, Moodie DS, et al. Difficulty in diagnosing double chambered right ventricle in adults. Am J Cardiol 2001;88:816-17.

2 McElhinney DB, Chatterjee KM, Reddy VM. Double-chambered right ventricle presenting in adulthood. Ann Thorac Surg 2000;70:124-5. 
3 Maron BJ, Ferrans VJ, White RI. Unusual evolution of acquired infundibular stenosis in patients with ventricular septal defect. Circulation 1973;48:1098.

4 Sanatani S, Peirone AR. Double-chambered right ventricle. eMed J 2001;2:7.

5 Singh M, Agarwala MK, Grover A, et al. Clinical, echocardiographic and angiographic profile of patients with double chambered right ventricle: experience with 48 cases. Angiology 1999;50:223-31.

6 Kilner PJ, Sievers B, Meyer GP, et al. Double-chambered right ventricle or subinfundibular stenosis assessed by cardiovascular magnetic resonance. J Cardiovasc Magn Reson 2002;4:373-9.

7 Restivo A, Cameron AH, Allwork SP. Divided right ventricle: a review of its anatomical varieties. Ped Cardiol 1984;5:197-204

8 Folger GM. Right ventricular outflow pouch associated with double chambered right ventricle. Am Heart J 1985; 109:1044-9.
9 Latson LA, Prieto LR. Right ventricular and pulmonary outflow abnormalities. In: Moss AJ, Adams FH, eds. Heart disease in infants, children and adolescents, 6th ed, vol 2. Philadelphia: Lippincott Williams and Wilkins, 0000:832-5.

10 Kirklin JW, Barratt-Boyes BG. Ventricular septal defect and pulmonary stenosis or atresia. In: Editor(s) names. Cardiac surgery, 2 nd ed, vol 2. New stenosis or atresia. In: Editor(s) names. Card
York: Churchill Livingstone, 1993:977-8.

11 Hoffman P, Stümper O, Rydlewska-Sadowska W, et al. Transgastric imaging - a valuable addition to the assessment of congenital heart disease. J Am Soc Echocardiogr 1993;6:35-44.

12 Sreeram N, de Bruijn D, Hitchcock JF. Double chambered right ventricle: delineation by multiplane transoesophageal echocardiography. Int J Cardiol 1998;66:309-11.

\section{IMAGES IN CARDIOLOGY}

doi: $10.1136 /$ hrt.2003.027748

\section{Emergency endovascular aortic stenting for the treatment of a ruptured atherosclerotic ulcer}

A 71 year old man presented with severe chest pain followed by collapse. On arrival he was hypotensive, tachycardic, and had a prominent left parasternal thrill. The chest $x$ ray revealed a widened mediastinum. His previous medical history included dialysis dependent chronic renal failure, mild aortic stenosis, hypertension, and asthma

Transthoracic and transoesophageal echocardiography demonstrated a large pseudoaneurysm of the descending aorta $(5.5 \mathrm{~cm}$ diameter) with an entry point just distal to the left subclavian artery (panel A). There was extensive mural and protuberant atheroma. Aortography confirmed the diagnosis of a ruptured penetrating aortic ulcer and the extension of the pseudoaneurysm (panel B). As the patient's operative risk was considered high he underwent emergency endovascular stenting using a $130 \mathrm{~mm}$ long, $34 \mathrm{~mm}$ diameter, covered stent-graft (Talent, Medtronic, Minneapolis, USA) (panels $\mathrm{C}$ and D). The patient made a good recovery and follow up scanning five days later revealed no flow into the aneurysm.

A penetrating atherosclerotic ulcer of the aorta is defined as an ulcerating atherosclerotic lesion that penetrates the elastic lamina. It is associated with haematoma formation within the media of the aortic wall and can lead to aortic dissection, aortic aneurysm, or rupture. The risk of aortic rupture in patients with penetrating ulcers $(40 \%)$ is significantly higher than in patients with classic type B dissection $(3.6 \%)$. Urgent intervention is required but emergency surgery is associated with mortality rates of up to $50 \%$. Endovascular stenting now offers an alternative treatment in such high risk patients.

J Eichhöfer

A R J Mitchell

A P Banning mitcharj@doctors.org.uk

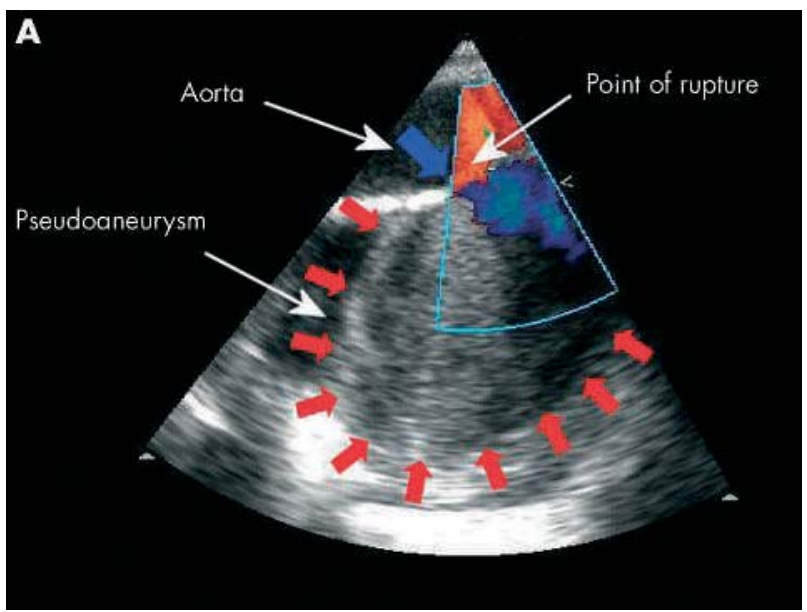

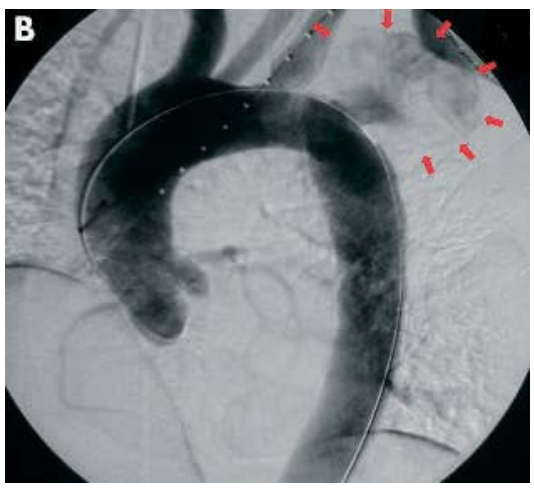
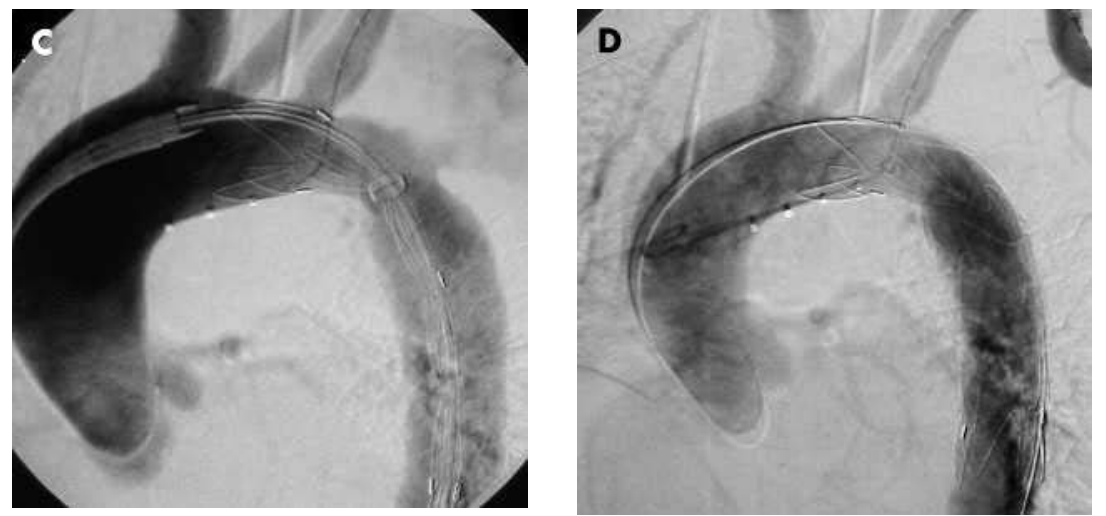